\title{
Delivery of propellant soluble drug from a metered dose inhaler
}

\author{
H L Ashworth, C G Wilson, E E Sims, P K Wotton, J G Hardy
}

\begin{abstract}
The deposition of particulate suspensions delivered from a metered dose inhaler has been investigated extensively. The distribution of propellant, delivered from a metered dose inhaler, was studied by radiolabelling it with technetium-99m hexamethylpropyleneamine oxime. Andersen sampler measurements indicated that half of the dose was associated with particles in the size range $0.5-5.5 m$ diameter. The preparation was administered to healthy subjects by inhalation and deposition was monitored with a gamma camera. Each lung image was divided into an inner, mid, and peripheral zone. The effects on deposition of varying the size of the delivery orifice $(0.46,0.61$, and $0.76 \mathrm{~mm}$ internal diameters) and the effect of attaching a spacer were assessed. Lung deposition was independent of the orifice size within the actuator. Without the spacer the average dose deposited in the lungs was $39 \%$, with $15 \%$ penetrating into the peripheral part of the lungs. Attachment of the spacer to the mouthpiece increased the mean lung deposition to $57 \%$ and reduced oropharyngeal deposition. The study has shown that propellant soluble drugs can be delivered efficiently to the lungs from a metered dose inhaler.
\end{abstract}

Metered dose inhalers provide a convenient means of delivering small doses of drugs to the lungs. They are widely used for the administration of bronchodilators, corticosteroids, and anticholinergic and anti-allergy compounds in the treatment of asthma.

Metered dose inhalers should be actuated during slow inhalation, followed by breath holding for 10 seconds, for maximum drug deposition in the lungs. For drug particles suspended in the propellant, up to $15 \%$ of the dose can be deposited in the lungs with this technique. ${ }^{1}$ Many patients, however, fail to achieve deposition of even $10 \% .^{2}$ Deposition can be improved by the attachment of a spacer to the actuator mouthpiece, ${ }^{3}$ the spacer providing a reservoir for the aerosol and reducing the need for precise synchronisation between actuation and inhalation. In addition, large aerosol particles tend to deposit in the spacer and reduce the amount of drug becoming impacted in the oropharynx. ${ }^{3}$

The deposition of particulate suspensions delivered from metered dose inhalers has been investigated extensively. Propellant soluble drugs such as the bronchodilator tulobuterol, however, may show different deposition patterns. We have monitored lung deposition of a compound that is soluble in the propellant administered from a metered dose inhaler to healthy subjects.

\section{Methods}

The metered dose inhaler canisters contained $10 \mathrm{ml}$ propellant and delivered $0.05 \mathrm{ml}$ per actuation. The propellant was a mixture of trichlorofluoromethane, dichlorotetrafluoroethane, and dichlorodifluoromethane plus sorbitan trioleate; it did not contain any drug. The mixture was radiolabelled with technetium$99 \mathrm{~m}$ hexamethylpropyleneamine oxime prepared by the addition of $5 \mathrm{GBq}{ }^{99 \mathrm{~m}} \mathrm{Tc}$ sodium pertechnetate in $5 \mathrm{ml}$ saline to a phial containing a freeze dried mixture of $0.5 \mathrm{mg}$ hexamethylpropyleneamine oxime, $7.6 \mu \mathrm{g}$ stannous chloride dihydrate, and $4.5 \mathrm{mg}$ sodium chloride (Amersham International, Amersham). The freshly prepared ${ }^{99 m} \mathrm{Tc}-$ hexámethylpropyleneamine oxime was added to a separating funnel containing $2 \mathrm{ml}$ trichlorofluoromethane. After the mixture had been shaken for two minutes the organic layer was separated and assayed for radioactivity. The solution was allowed to evaporate at room temperature until the concentration was 1-2 $\mathrm{GBq} / \mathrm{ml}$. To an empty canister, cooled in liquid nitrogen, was added $0.3 \mathrm{ml}$ radiolabelled trichlorofluoromethane and $10 \mathrm{ml}$ propellant mixture from a precooled canister. After a metering valve had been fitted the canister was allowed to warm to room temperature and the contents were assayed for radioactivity. Typically, 2-3 MBq ${ }^{99 m} \mathrm{Tc}$ labelled propellant was delivered per actuation.

Aerosol delivery was compared by using actuators with internal orifice diameters of 0.46 , 0.61 , and $0.76 \mathrm{~mm}$. Aerosol deposition from the actuator with the $0.61 \mathrm{~mm}$ diameter orifice was also compared with and without the addition of a $10 \mathrm{~cm}$ long, $45 \mathrm{~cm}^{3}$ prototype commercial spacer (without a valve) to the mouthpiece. The dose distributions from each delivery system were assessed by using an Andersen eight stage sampler. Tén doses of radiolabelled propellant were fired into the sampler at a rate of one dose every 15 seconds. The apparatus was then dismantled and each component, including the actuator, was assayed for radioactivity with a probe scintillation detector.

Aerosol doses were administered to six healthy subjects aged 18-25 years. All were 
Aerosol distribution following administration to volunteers

\begin{tabular}{|c|c|c|c|c|}
\hline \multirow{2}{*}{$\begin{array}{l}\text { Deposition } \\
\text { site }\end{array}$} & \multicolumn{4}{|c|}{ Proportion of radioactivity (mean $(S D) \%$ ) for orifice diameter ( $\mathrm{mm}$ ) } \\
\hline & 0.46 & 0.61 & $0 \cdot 76$ & 0.61 (plus spacer) \\
\hline Actuator & 21 & 15 & 17 & 32 \\
\hline Oropharynx & $39(9)$ & $47(8)$ & $45(22)$ & $11(5)$ \\
\hline Lung - total & $40(15)$ & $38(10)$ & $38(15)$ & $57(25)$ \\
\hline \multicolumn{5}{|l|}{ Lung-regions } \\
\hline Inner zone & $11(3)$ & $13(4)$ & $12(4)$ & $18(7)$ \\
\hline Mid zone & $11(8)$ & $11(3)$ & $12(4)$ & $16(7)$ \\
\hline Peripheral zone & $18(15)$ & $14(4)$ & $14(7)$ & $23(11)$ \\
\hline
\end{tabular}

non-smokers and had a forced expiratory volume in one second $\left(\mathrm{FEV}_{1}\right)$ of $80-147 \%$ predicted. The lung dimensions of each subject were determined from anterior and posterior krypton- $81 \mathrm{~m}$ ventilation images recorded with a gamma camera. The study was approved by the local ethical committee and the administration of the radiopharmaceutical authorised by the Department of Health. Each subject provided written informed consent.

Subjects received the radiolabelled aerosol from each delivery system on separate occasions in a randomised order. On each occasion, with the subject standing, the lips were closed around the mouthpiece and the aerosol was delivered at the start of a slow, deep inhalation followed by 10 seconds' breath holding. Two to four shots were administered to deliver about $2 \mathrm{MBq}{ }^{99 \mathrm{~m}} \mathrm{Tc}$ into the lungs. Anterior and posterior images of the lungs and stomach and a lateral view of the head and neck were then.recorded with a gamma camera. Each image was of 400 seconds' duration and was stored by computer.

The images were displayed on a television monitor and regions of interest defined. Count rates were determined for the whole of the lungs, including the trachea and carina, stomach, lower oesophagus, and oropharynx.
The distribution of the radioactivity within the lungs was quantified according to the method of Agnew and coworkers. ${ }^{4}$ The krypton images were used to define the lung shapes. The shape of each lung was considered in terms of a matrix 5 units wide by 8 units high. For each lung an inner zone comprising six cells and a peripheral zone of 21 cells were defined. Count rates from the inner zones plus the trachea and carina would be predominantly from radioactivity deposited in the large airways and those from the peripheral zones mainly from radioactivity in the small conducting airways and alveoli. Each count rate was corrected for background counts and the geometric means of corresponding anterior and posterior count rates were calculated to compensate for gamma ray attenuation.

\section{Results}

The distribution of the radioactivity within the Andersen sampler indicated that about half of the dose was associated with particles with a diameter in the range $0.5-5 \mu \mathrm{m}$, with more than $40 \%$ in the range $1-4 \mu \mathrm{m}$. This was not affected by the size of the aerosol delivery orifice within the actuator. The spacer reduced the proportion of the dose deposited in the throat of the apparatus from $24 \%$ to $1 \%$. On average, $18 \%$ of the dose was retained in the actuator (table).

The ${ }^{81 \mathrm{~m}} \mathrm{Kr}$ images showed a normal pattern of lung ventilation in all subjects. The regions of interest over the inner zones, including the trachea and carina, represented a mean (SD) of $26 \%(1.2 \%)$ of the area of the lung images and those over the peripheral zones $51 \%(1.8 \%)$. In terms of the ${ }^{81 \mathrm{~m}} \mathrm{Kr}$ distribution, the count rates from these zones were $26 \%(1.6 \%)$ and $47 \%$ $(1.9 \%)$ respectively of the count rates from the whole lungs.

The distributions of the radioactivity after aerosol administration to the volunteers are
Posterior images showing aerosol deposition in the lungs and swallowed tracer in the stomach after inhalation (a) without and (b) with the spacer attachment.

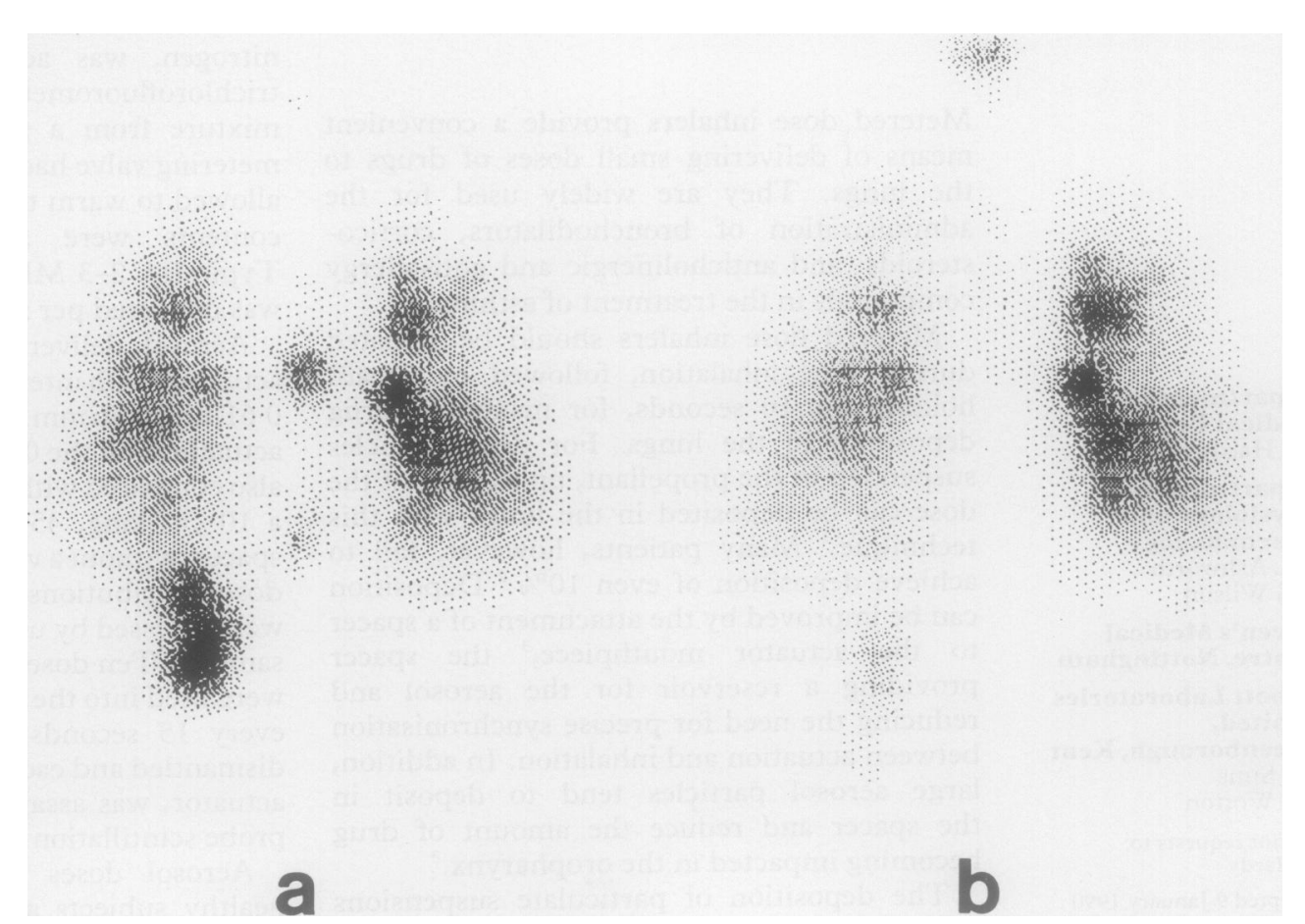


shown in the table. A mean of $39 \%$ of the dose was deposited in the lungs and the percentage did not depend on the orifice size of the actuator. Within the lungs, on average, $31 \%$ was deposited in the inner zone and $40 \%$ in the peripheral zone, representing less peripheral penetration than was observed with the ${ }^{81 \mathrm{~m}} \mathrm{Kr}$ gas. The tendency for the aerosol to be deposited more centrally is illustrated in the figure. The effect of the spacer was to increase the lung deposition and to reduce the proportion becoming impacted in the oropharynx and subsequently swallowed (figure). No tracer was detected in the region of the heart or liver, indicating that there was little absorption from the lungs into the blood during imaging.

\section{Discussion}

Particle size is a major factor influencing the deposition of inhaled aerosols. ${ }^{5}$ Particles with a diameter greater than $8 \mu \mathrm{m}$ are impacted mainly in the oropharynx, whereas those in the range 1-8 $\mu \mathrm{m}$ tend to be deposited in the conducting airways and alveoli. The proportion of particles deposited by inertial impaction in the airways increases with particle size and flow rate. Deposition by gravitational sedimentation increases as the air flow velocity decreases, so that a slow inhalation followed by breath holding at total lung capacity maximises aerosol deposition in the lungs. Aerosol particles may change in size during inhalation. Hygroscopic particles may increase in size, and chlorofluorocarbon propellant particles will tend to evaporate. The consistency of the aerosol deposition patterns from actuators with different orifice diameters suggests that the initial particle size of the aerosol spray is not a dominant factor influencing lung deposition. The size distributions determined with the Andersen sampler indicated that most of the inhaled particles were of an optimum size for deposition in the lungs.

Drug particles-for example, terbutaline sulphate crystals-suspended in the propellant of metered dose inhalers have an aerodynamic mass median diameter of about $3 \mu \mathrm{m}$. Drug delivery to the lungs can be assessed by introducing radiolabelled solid particles of similar size into the formulation and monitoring the deposition. ${ }^{2}$ Even with good synchronisation between actuation of the device and inhalation followed by breath holding lung deposition is unlikely to exceed $15 \% .^{1}$ In the present study on average $39 \%$ of the soluble tracer was deposited in the lungs. This relatively high deposition is likely to have resulted from evaporation of the propellant droplets during inhalation. The fact that similar deposition patterns were seen with actuators having different orifice diameters suggests that the change in particle size due to evaporation is of overriding importance. The use of the spacer would have increased the time available for droplet evaporation, thereby enhancing lung deposition. The advantage of small particle size is exemplified by the use of ${ }^{99 \mathrm{~m}} \mathrm{Tc}$ labelled carbon particles, $5.0 \mathrm{~nm}$ and less, to provide diagnostic lung images comparable to those obtained by breathing radioactive gases. ${ }^{6}$

In addition to increasing lung deposition attachment of the spacer reduced oropharyngeal deposition. Thus the use of the spacer has a dual role-enhancing drug delivery to the lungs while helping to protect the throat from any irritant effects of the drug. ${ }^{1}$ The present results were obtained with healthy subjects, trained in the use of metered dose inhalers. As with drug suspensions delivered by metered dose inhaler, the lung deposition of drugs that are soluble in the propellant is likely to be reduced in patients who fail to synchronise actuation of the device with inhalation.

Bronchodilator drugs, such as tulobuterol, ${ }^{7}$ administered from metered dose inhalers for the treatment of asthma and chronic bronchitis exert their therapeutic effects by interaction

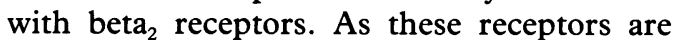
widespread throughout the airway epithelium, ${ }^{8}$ good penetration of the drug to the smaller airways may be beneficial. The results of the present study indicate that this can be achieved with propellant soluble drugs delivered from a metered dose inhaler. Thus solution aerosols offer good potential for efficient drug delivery by inhalation.

We wish to thank Kavita Dosanjh and Robert Honeysett for advice concerning the particle sizing.

1 Newman S, Clarke SW. Therapeutic aerosols 1-physical and practical considerations. Thorax 1983;38:881-6.

2 Newman S, Pavia D, Moren F, Sheahan NF, Clarke SW. Deposition of pressurised aerosols in the human respiratory tract. Thorax 1981;36:52-5.

3 Newman SP, Millar AB, Lennard-Jones TR, Moren F, Clarke SW. Improvement of pressurised aerosol deposition with Nebuhaler spacer device. Thorax 1984;39: 935-41.

4 Agnew JE, Francis RA, Pavia D, Clarke SW. Quantitative comparison of ${ }^{99 m} \mathrm{Tc}$-aerosol and ${ }^{81} \mathrm{Kr}^{\mathrm{m}}$ ventilation images. Clin Phys Physiol Meas 1982;3:21-30.

5 Morrow PE. Aerosol characterization and deposition. Am Rev Respir Dis 1974;110(suppl):88-99.

6 Burch WM, Sullivan PJ, McLaren CJ. Technegas-a new ventilation agent for lung scanning. Nucl Med Commun 1986;7:865-71.

7 Patel KR. Bronchodilator activity of a new inhaled $\beta_{2}-$ adrenoceptor agonist, tulobuterol and its protective effect in exercise-induced asthma. Br J Clin Pharmacol 1986; in exercise-ir $21: 234-7$.

8 Carstairs JR, Nimmo AJ, Barnes PJ. Autoradiographic visualization of beta-adrenoceptor subtypes in human lung. Am Rev Respir Dis 1985;132:541-7. 